\title{
MEDICAMENTOS GENÉRICOS: UMA ABORDAGEM NO MUNICÍPIO DE VALE DO PARAÍSO, RONDÔNIA
}

\author{
Cleusa Moura da SILVA, Tiago Barcelos VALIATTI*, Richard da Silva Pereira CALAZANS, Izabel \\ Bárbara BARCELOS \& Jeferson de Oliveira SALVI
}

Centro Universitário Luterano de Ji-Paraná. Ji-Paraná, Rondônia, Brasil.

*Autor para correspondência: tiago_valiatti@ hotmail.com

DOI: http://dx.doi.org/10.18571/acbm.142

\section{RESUMO}

O objetivo do presente estudo foi avaliar o perfil de utilização dos medicamentos genéricos pela população do município de Vale do Paraíso, estado de Rondônia. Para tanto, foram entrevistados 368 moradores da cidade por meio da aplicação de um questionário. Excluiu-se 11 questionários devido ao preenchimento incorreto, totalizando uma amostra final de 357 indivíduos, onde $62 \%$ eram do sexo feminino e $38 \%$ do sexo masculino. Quanto ao grau de escolaridade o mais frequente entre os entrevistados foi o nível médio, e $93 \%$ afirmaram possuir renda mensal de até 5 salários mínimos. Do total de participantes, $76 \%$ relataram já ter utilizado medicamentos genéricos, sendo que maioria os obtém com receita médica e alcançaram o resultado desejado, após sua utilização. Constatou-se que para $77 \%$ dos participantes o valor dos genéricos influencia no momento da compra, sendo este fato mais comum entre os homens. Além do mais, verificou-se que maioria dos entrevistaram relataram que médicos e farmacêuticos fazem referência aos medicamentos genéricos, e que confiam no profissional farmacêutico para realizar a troca dos medicamentos de referência para genéricos. Os resultados demonstraram uma boa aceitação dos genéricos por parte dos entrevistados, visto que ampla maioria já fez o uso deste.

Palavras-chaves: Genéricos; Legislação; Mercado Farmacêutico.

\begin{abstract}
The objective of the present study was to evaluate the profile of the use of generic drugs by the population of the municipality of Vale do Paraíso, state of Rondônia. For that, 368 residents of the city were interviewed through the application of a questionnaire. Eleven questionnaires were excluded due to incorrect completion, totaling a final sample of 357 individuals, where $62 \%$ were female and 38\% male. Regarding the level of education, the most frequent among the interviewees was the average level, and $93 \%$ reported having a monthly income of up to 5 minimum wages. Of the total number of participants, $76 \%$ reported having already used generic drugs, most of them receiving a prescription and achieving the desired result after their use. It was found that for $77 \%$ of the participants, the value of generics influences the moment of purchase, being this fact more common among men. Furthermore, it was found that most of the interviewees reported that doctors and pharmacists refer to generic medicines, and that they rely on the pharmaceutical professional to carry out the exchange of reference medicines for generics. The results showed a good acceptance of the generics by the interviewees, since a large majority has already made use of this one.
\end{abstract}

Keywords: Generic; Legislation; Pharmaceutical Market. 


\section{Introdução}

Medicamentos são produtos farmacêuticos que apresentam finalidade profilática, curativa, paliativa ou para fins de diagnóstico, sendo, portanto, importantes ferramentas para melhorar e manter a saúde (BRASIL, 2003). A dificuldade de regulação do mercado farmacêutico e os elevados custos dificultam o acesso da população aos medicamentos, estima-se que até um terço da população apresente alguma dificuldade em acessá-los (WHO, 2004). Devido a esse fato, os medicamentos genéricos vêm sendo utilizados em diversos países como agentes reguladores desse amplo mercado, já que exerce papel direto na oferta e demanda (CARVALHO, ACCIOLY e RAFFIN, 2006).

No mercado farmacêutico comandado pelas grandes indústrias, os medicamentos comercializados são classificados em três principais categorias: os de referência, os genéricos e os similares (SILVA e ROCHA, 2016). De acordo com a legislação brasileira, o medicamento de referência é inovador, que tem sua eficácia e segurança comprovada através de ensaios clínicos, como a biodisponibilidade que é determinada durante o desenvolvimento do produto, antes mesmo de ter a obtenção do registro para comercialização (BRASIL, 1999).

Os similares são aqueles que possuem os mesmos princípios ativos, nas mesmas concentrações, forma farmacêutica, via de administração que o medicamento de referência, podendo diferir em características como tamanho e forma do produto, embalagem, excipientes e veículos, nome comercial e outros (BRASIL, 1999).

Já os genéricos, instituídos através da Lei no 9.787 implantada em 10 de fevereiro de 1999, são medicamentos que além de possuírem as mesmas semelhanças dos similares perante aos de referência, são intercambiáveis a estes, e devem ser submetidos os mesmos testes de qualidade, eficácia e bioequivalência (mesma biodisponibilidade) (SILVA e ROCHA, 2016). Este tipo de medicamento deve ser identificado apenas pela nomenclatura de seu(s) principio(s) ativo(s), não podendo deter de nome comercial ou fictício, além de dever possuir em sua embalagem, uma tarja amarela com a letra “G” em destaque, para fácil percepção (BRASIL, 1999).

Logo, na sua produção a isenção de gastos em pesquisas e publicidades para as indústrias farmacêuticas proporcionam na maioria das vezes, sua comercialização com preços menores quando se comparado com as outras duas categorias, se enquadrando uma alternativa de aos de referências, dos quais vários são importados e encarecidos no país (VIEIRA E ZUCCHI, 2006). O que corrobora para o fato de o preço comercial destes produtos terem sido de até $35,5 \%$ mais baratos em 2014, participando cada vez mais no comércio brasileiro (BERTOLDIL, 2016).

Portanto, os medicamentos genéricos se diferenciam dos medicamentos de referência somente pela nomenclatura, pois levam o nome do princípio ativo seguido pela letra G maiúscula, na embalagem. A nomenclatura é designada conforme a Denominação Comum Brasileira (DCB) ou, na ausência da mesma, conforme a Denominação Comum Internacional (DCI) (BLAT et al., 2012; FERNANDES et al., 2011).

No âmbito do Sistema Único de Saúde (SUS), o profissional prescritor deverá obrigatoriamente utilizar a DCB ou, não havendo a mesma, deverá adotar a DCI no ato da prescrição, entretanto, nas redes privadas de saúde fica a critério do prescritor a utilização do nome comercial ou dos genéricos, podendo o mesmo fazer observações com restrição à intercambialidade (BRASIL, 2003).

Apesar da desconfiança de uma considerável parcela de prescritores, a utilização dos medicamentos genéricos tem crescimento de modo acelerado no Brasil, passados mais de 15 anos da sua inserção no mercado, o uso desta categoria já atingiu cerca de $45,5 \%$ da população brasileira, sendo aproximadamente um terço dos medicamentos utilizados neste período (BERTOLDIL, 2016). Uma pesquisa revela que já ocupa a $6^{a}$ posição no mercado mundial farmacêutico e que este chegará a 4 ${ }^{a}$ posição em 2017 (IMS Health, 2013).

Diante disso, o presente trabalho teve como objetivo avaliar o perfil de utilização dos 
medicamentos genéricos no município de Vale do Paraíso, Rondônia.

\section{Metodologia}

Foi desenvolvido um estudo transversal, por meio do levantamento de dados, no município de Vale do Paraíso, na região leste do estado de Rondônia. Para determinação da amostra, utilizou-se a ferramenta online de Santos (2016) levando em consideração a população do município que em 2010 era de 8.231 habitantes de acordo com o Instituto Brasileiro de Geografia e Estatística (IBGE), totalizando 368 indivíduos a serem entrevistados.

A pesquisa foi desenvolvida junto ao projeto: Medicamentos Genéricos: aspectos após 15 anos de vigência da lei 9.787/99 na região centro oeste de Rondônia, aprovado pelo Comitê de Ética e Pesquisa (CEP) do Centro Universitário Luterano de Ji-Paraná por meio do parecer $\mathrm{n}^{\circ}$. 558.11812015.

O questionário utilizado investigou aspectos sociodemográficos abordando as seguintes variáveis: escolaridade do usuário, renda familiar, frequência de compra de medicamentos genéricos, satisfação com relação ao medicamento genérico, grau de confiança, o questionamento do cliente com o médico no momento da prescrição médica, obtenção de prescrição do medicamento genérico, obtenção de informações sobre o medicamento genérico por parte do farmacêutico, relevância do preço no momento da compra do medicamento e confiança no farmacêutico.

Os dados foram tabulados com auxílio do programa Microsoft Excel (2010®) e interpretados por meio de estatística descritiva simples, considerando a frequência relativa para os eventos. Os dados não paramétricos para as possibilidades de respostas foram comparados de maneira pareada entre os gêneros por meio do teste de Wilcoxon e a associação entre as variáveis dependentes e independentes analisada pelo teste do Qi quadrado de Student, considerando o nível de significância de 95\%, com auxílio do software GraphPad Prism ${ }^{\circledR}$ (versão 6.0)

\section{Resultados}

Do total de entrevistados, 11 questionários foram excluídos por preenchimento incorreto, obtendo assim, uma amostra final de 357 indivíduos. Dentre os participantes 220 pessoas eram do sexo feminino (60\%) e 137 eram do sexo masculino (37\%). Conforme demonstrado na tabela 1 , a maioria $(33 \%)$ dos participantes possui nível médio, sendo mais frequente entre as mulheres.

Tabela 1: Grau de escolaridade estratificado por sexo. Vale do Paraíso, Rondônia.

\begin{tabular}{ccccccc}
\hline \multirow{2}{*}{ Escolaridade } & \multicolumn{2}{c}{ Masculino } & \multicolumn{2}{c}{ Feminino } & \multicolumn{2}{c}{ Total } \\
\cline { 2 - 7 } & $\mathbf{n}$ & $\mathbf{\%}$ & $\mathbf{n}$ & $\mathbf{\%}$ & $\mathbf{n}$ & $\mathbf{\%}$ \\
\hline Somente sei ler & 52 & $38 \%$ & 55 & $25 \%$ & 107 & $30 \%$ \\
Nível fundamental & 33 & $24 \%$ & 62 & $28 \%$ & 95 & $26 \%$ \\
Nível médio & 41 & $30 \%$ & 76 & $35 \%$ & 117 & $33 \%$ \\
Nível superior & 8 & $6 \%$ & 16 & $7 \%$ & 24 & $7 \%$ \\
Pós-graduado & 4 & $3 \%$ & 10 & $5 \%$ & 14 & $4 \%$ \\
\hline
\end{tabular}

Os valores encontrados não diferiram significativamente entre si, como também, não houve uma associação entre as variáveis.

Com relação à renda, $93 \%$ dos entrevistados afirmaram possuir rendimento mensal de até 5 salários, $6 \%$ de 5 a 10 salários e $1 \%$ relataram ganhar mais de 10 salários mínimos.

A Tabela 2 demonstra que a minoria dos entrevistados utiliza medicamentos de forma contínua, e que uma grande parcela dos indivíduos estudados já utilizou medicamentos genéricos. 
Quase um quarto dos entrevistados usaram a receita médica para obter os medicamentos genéricos. Ainda, os participantes relataram em sua maioria, que obtiveram os resultados esperados, além de que durante a consulta são informados a respeito dos genéricos, e que o valor influencia na compra destes.

Quando indagados se o profissional farmacêutico faz menção ao medicamento genérico $69 \%$ dos candidatos responderam de forma afirmativa e pouco mais de $60 \%$ relataram confiar no profissional farmacêutico.

Tabela 2: Perfil de utilização de medicamentos genéricos. Vale do Paraíso, Rondônia.

\begin{tabular}{|c|c|c|c|c|c|c|}
\hline \multirow{2}{*}{ Variáveis } & \multicolumn{2}{|c|}{ Masculino } & \multicolumn{2}{|c|}{ Feminino } & \multicolumn{2}{|c|}{ Total } \\
\hline & Sim & Não & Sim & Não & Sim & Não \\
\hline Utiliza medicamento de forma contínua? & $39 \%$ & $61 \%$ & $31 \%$ & $69 \%$ & $34 \%$ & $66 \%$ \\
\hline Já utilizou medicamento genérico? & $78 \%$ & $22 \%$ & $75 \%$ & $25 \%$ & $76 \%$ & $24 \%$ \\
\hline $\begin{array}{l}\text { Obteve o medicamento genérico com receita } \\
\text { médica? }\end{array}$ & $75 \%$ & $25 \%$ & $70 \%$ & $30 \%$ & $72 \%$ & $28 \%$ \\
\hline Obteve o resultado desejado? & $67 \%$ & $33 \%$ & $83 \%$ & $17 \%$ & $77 \%$ & $23 \%$ \\
\hline $\begin{array}{l}\text { O valor exerce influencia na compra do } \\
\text { medicamento? }\end{array}$ & $89 \%$ & $11 \%$ & $66 \%$ & $44 \%$ & $75 \%$ & $25 \%$ \\
\hline $\begin{array}{l}\text { Em consulta médica é informado sobre o } \\
\text { medicamento? }\end{array}$ & $56 \%$ & $44 \%$ & $61 \%$ & $39 \%$ & $59 \%$ & $41 \%$ \\
\hline $\begin{array}{l}\text { O farmacêutico faz referência ao medicamento } \\
\text { genérico? }\end{array}$ & $70 \%$ & $30 \%$ & $68 \%$ & $32 \%$ & $69 \%$ & $31 \%$ \\
\hline $\begin{array}{l}\text { Você confia no farmacêutico que faz a troca para o } \\
\text { medicamento genérico? }\end{array}$ & $65 \%$ & $35 \%$ & $61 \%$ & $39 \%$ & $62 \%$ & $38 \%$ \\
\hline
\end{tabular}

A análise pareada dos dados por gênero, através do teste de Wilcoxon de maneira pareada, indicou diferença significativa entre as possibilidades de respostas para os homens $(p=0,234)$, bem como, uma associação entre as variáveis por meio do teste do Qi quadrado de Fisher ( $\mathrm{p}<0,0001)$. Para as mulheres, as opções de resposta não foram significativamente distintas entre si, porém, se observou a mesma a associação entre as variáveis dependentes $(\mathrm{p}<0,001)$.

\section{Discussão}

Os dados obtidos revelaram que os medicamentos genéricos são utilizados de forma contínua por aproximadamente um terço dos participantes entrevistados. Em estudo semelhante, Fernandes et al (2011) constataram valor superior ao registrado no presente estudo, visto que 58\% declararam utilizar medicamentos de forma contínua.

Fatores como responsabilidade excessiva, ocorrência de situações estressantes, falta de segurança e de estabilidade no emprego, carga horária intensa, trabalhos domésticos, maus hábitos alimentares, entre outros, estão associados diretamente a uma piora na saúde da população, originando, portanto, diversas doenças e agravos à saúde que acaba causando o uso contínuo de medicamentos (SANTOS e NITRINI, 2004; HOLMGREN et al., 2009; PEREIRA et al., 2011).

Evidenciou-se que $76 \%$ dos entrevistados já adquiriram os medicamentos genéricos pelo menos uma vez na vida, revelando, uma grande aceitação com relação a esses medicamentos, indicando ainda, que há acessibilidade a estes. Utzig et al (2009), na cidade de Toledo (PR), verificaram resultados superiores aos aqui encontrados, pois $98 \%$ dos participantes da pesquisa já adquiriram em algum momento da vida os medicamentos genéricos. Já Oliveira et al. (2005) registraram um percentual de $70 \%$ de utilização de genéricos, sendo, portanto, dados semelhantes aos aqui encontrado.

De acordo com Bertoldi et al (2014) no decorrer dos anos, a resistência inicial observada logo após a implantação dos medicamentos genéricos foi acabando, frente às vantagens oferecidas 
por estes. Guttier et al (2016) comprovam esse fato em seu estudo, onde se verificou a utilização de genéricos na cidade de Pelotas RS, em 2002 e 2012, sendo observado um aumento substancial desses medicamentos.

Ainda, Bertoldi et al. (2014) relatam a presença dos genéricos em cerca de $30 \%$ dos grupos farmacológicos de alta procura no mercado, o que também contribui para o aumento da sua utilização.

Constatou-se que maioria dos participantes adquiriram os medicamentos genéricos com receita médica, no entanto, não significa que nestas receitas estavam prescritos os genéricos, pois no momento da compra o farmacêutico pode ter feito a intercambialidade entre o medicamento de referência com o genérico.

Em estudos realizados, tem se observado certa resistência por parte dos prescritores com relação aos medicamentos genéricos. Pacientes estudados por Blatt et al. (2012) relataram que $34,6 \%$ dos médicos que costumam se consultar não prescrevem o genérico, enquanto apenas 23,5\% prescrevem frequentemente. Já resultados descritos por Lira et al. (2014) mostram que $17,6 \%$ dos médicos com os quais os participantes da pesquisa se tratavam, nunca tinham prescrito o genérico, sendo que os profissionais que prescreviam frequentemente representaram apenas $7,5 \%$.

Estudos mostram que ampliar o conhecimento dos prescritores, a respeito desses medicamentos, pode ser uma boa medida para aumentar o número da prescrição (HEIKKILÃ, MÃNTYSELKÃ e AHONEN, 2011; VALLÉS et al., 2003).

O questionamento sobre o resultado desejado teve o intuito de avaliar a satisfação do usuário mediante a auto-observação. Para a investigação sobre o alcance do resultado desejado, após o tratamento com medicamentos genéricos, revelou que $77 \%$ responderam positivamente, sendo este resultado inferior ao evidenciado no estudo de Fernandes et al. (2011), onde 84\% concordaram com a indagação.

Ressalta-se que obrigatoriamente os medicamentos genéricos durante seu registro são submetidos a um rígido controle de qualidade, passando pelos testes de bioequivalência e biodisponibilidade, a fim de garantir as mesmas características do medicamento de referência, portanto, os indivíduos que relataram não ter obtido resultados satisfatórios podem ter sido influenciados por outros fatores, como por exemplo, a não adesão ao tratamento, e não pela baixa qualidade do genérico utilizado (BRASIL, 2003; WHO, 2003).

No presente estudo a maioria absoluta dos homens relatou que o valor do medicamento influencia no momento da compra, já as mulheres se preocuparam menos, evidenciando assim, que o valor é fator determinante na aquisição dos genéricos. Além do mais, ressalta-se, que a diferença observada entre homens e mulheres pode estar relacionada às características da própria região estudada, onde em grande parte dos lares os homens são os responsáveis pelo sustento, buscando sempre por meios mais econômicos. Resultados semelhantes foram encontrados por Lira et al. (2014) onde constataram que o preço é fundamental no momento da compra dos genéricos, visto que $80,2 \%$ dos participantes de sua pesquisa relataram comprar medicamentos genéricos apenas por conta do preço.

Segundo resolução da Câmera de Regulação do Mercado de Medicamentos (CMED) o preço dos genéricos deve ser no mínimo $35 \%$ menor do que o produto de referência, contudo em uma pesquisa realizada pelo Instituto Brasileiro de Defesa do Consumidor (IDEC) foram identificados genéricos com preço superior ao de referência (IDEC, 2013). O instituto verificou ainda uma grande variação no preço dos genéricos entre as principais redes de drogarias do estado de São Paulo, sendo relatado que o controle dos preços dos medicamentos tem sido feito pelos próprios laboratórios e não pelo governo (IDEC, 2012).

No presente estudo observou-se que os farmacêuticos foram mais citados que os médicos, quando questionados se estes profissionais faziam referência aos genéricos. Estudo semelhante realizado por Marco (2013) revelou que 52\% das pessoas recebem informações sobre 
medicamentos genéricos, e destes, 58\% declararam ter vindo de um profissional farmacêutico, enquanto que apenas $20 \%$ relacionaram à consulta médica. Portanto, é possível refletir sobre a importância do profissional farmacêutico na divulgação dos medicamentos genéricos, principalmente na prestação de esclarecimentos no momento da compra (OLIVEIRA et al, 2005; MIRANDA et al., 2010).

Perguntou-se aos participantes se eles confiam no farmacêutico que fazem a troca dos genéricos, onde, 65 dos homens e $61 \%$ das mulheres responderam de forma positiva. Destaca-se que o profissional farmacêutico é o único capacitado para efetuar a intercambialidade entre dois medicamentos, ou seja, a substituição do medicamento de referência por seu genérico correspondente, desde que não exista restrição por parte do prescritor (BRASIL, 2003).

No entanto, salienta-se que somente pode realizar a troca do medicamento de referência por seu genérico, sendo proibida a substituição pelos medicamentos similares (BRASIL, 2003). De acordo com Vieira e Zucchi (2006), os laboratórios farmacêuticos geralmente adotam um sistema de bonificações e as drogarias um sistema de comissionamento pela venda de produtos similares, fato esse, que tem contribuído para o aumento das indicações de medicamentos similares no lugar dos medicamentos genéricos.

Nesse contexto, ressalta-se o desafio atemporal para a política dos genéricos, pois se percebeu a necessidade de educação contínua acerca deste assunto para os profissionais responsáveis por estimularem o uso desses medicamentos. Acredita-se que o profissional farmacêutico juntamente com o médico devem ser os maiores estimuladores dos medicamentos genéricos, buscando sempre passar informações acerca destes para os pacientes, pois na maioria das vezes são pessoas leigas com pouco ou nenhum entendimento do assunto, tomando, portanto, suas decisões sobre qual medicamento comprar, diante da orientação de um profissional capacitado (SILVA et al., 2013).

\section{Conclusão}

Conclui-se que grande parte dos entrevistados já utilizaram e alcançaram o resultado desejado por meio dos medicamentos genéricos, evidenciando boa aceitação dos mesmos. Além do mais, o valor desses medicamentos influenciou diretamente em sua compra. Ainda, médicos e farmacêuticos estão fazendo referência aos genéricos, conforme relato pela maioria dos participantes, que também confiam no farmacêutico para realizar a intercambialidade entre os medicamentos de referência e genéricos.

\section{Referências}

BERTOLDI, A. D. et al. Utilização de medicamentos genéricos na população brasileira: uma avaliação da PNAUM 2014. Revista de Saúde Pública. v.50(supl 2):11s, 2016.

BLATT, C.R. et al. Conhecimento popular e utilização dos medicamentos genéricos na população do município de Tubarão, SC. Ciência \& Saúde Coletiva. v.17, n.1, p.79-87, 2012.

BRASIL. Lei $\mathbf{N}^{\circ}$ 9.787, de 10 de fevereiro de 1999. Altera a Lei ${ }^{\circ} 6.360$, de 23 de setembro de 1976, que dispõe sobre a vigilância sanitária, estabelece o medicamento genérico, dispõe sobre a utilização de nomes genéricos em produtos farmacêuticos e dá outras providências. Diário Oficial da União 1999.

BRASIL. Ministério da Saúde. Resolução - RDC no 135, de 29 de maio de 2003. Regulamento técnico para medicamentos genéricos. Diário Oficial da União. Brasília, 2003. 
CARVALHO, M.C.R.D.; ACCIOLY, J.R.H.; RAFFIN, F.N. Representações sociais do medicamento genérico por consumidores residentes em Natal, Rio Grande do Norte, Brasil. Cadernos de Saúde Pública. v.22, n.3, p.653-661, 2006.

FERNANDES, E.A.F.; GONÇALVES, D.C.; SIMEONI, L.A.; SILVEIRA, D.; MELLO, M.H. Facetas da prescrição de medicamentos do Brasil: genérico, similar, referênciae intercambialidade. Brasília médica. v.48, n.2, p.188-194, 2011.

GUTTIER, M.C.; SILVEIRA, M.P.T.; LUIZA, V.L.; BERTOLSI, A.D. Percepção, conhecimento e uso de medicamentos genéricos no Sul do Brasil: o que mudou entre 2002 e 2012?. Cadernos de Saúde Pública. v.32, n.7, p. 1-13, 2016.

HEIKKILÄ, R.; MÄNTYSELKÄ, P.; AHONEN, R. Do people regard cheaper medicines effective? Population survey on public opinion of generic substitution in Finland. Pharmacoepidemiol Drug Saf . v.20, n.2, p.185-191, 2011.

HOLMGREN, K.; DAHLIN-IVANOFF, S.; BJORKELUND, C.; HENSING, G. The prevalence of work-related stress, and its association with self-perceived health and sick-leave, in a population of employed Swedish women. BMC Public Health. v.9, n73, 2009.

IMS Health (2013). The Global Use of Medicines: Outlook through 2017. The Global Use of Medicines: Outlook through 2017 (pp. 42). Estados Unidos.

Instituto Brasileiro de Defesa do Consumidor (IDEC). Diferença além da conta. Revista do

IDEC. 2012. Disponível em: < http://www.idec.org.br/uploads/revistas_materias/pdfs/ed-162capa.pdf> Acessado em: 15 de maio de 2017.

Instituto Brasileiro de Defesa do Consumidor (IDEC). Idec constata que preço de medicamentos sobe acima da inflação em quatro anos. 2013. Disponível em: http://www.idec.org.br/emacao/em-foco/idec-constata-que-preco-de-medicamentos-sobe-acima-da-inflaco-em-quatro-anos Acessado em: 15 de maio de 2017.

LIRA, C.A.B.; OLIVEIRA, J.N.S.; ANDRADE, M.S.; VANCINI-CAMPANHARO, C.R.; VANCINI, R.L.V. Knowledge, perceptions and use of generic drugs: a cross sectional study. Einstein. v.12, n.3, p.267-273, 2014.

MARCO, T. de. Verificação do grau de aceitação de medicamentos genéricos em uma farmácia de médio porte situada no Sul de Santa Catarina. Trabalho de Conclusão de Curso. (Graduação em Farmácia) - Universidade do Extremo Sul Catarinense, UNESC, Criciuma, SC, 2013.

MIRANDA, G.S.; GONÇALVES, E.F.; DUARTE, M.S.L.; CARVALHO, M.L.; CARVALHO, C. A. Avaliação do conhecimento da população de Viçosa-MG acerca dos medicamentos genéricos. Revista Eletrônica de Farmácia. v.7, n.3, p.34-43, 2010.

OLIVEIRA, S.F. et al: Prevalência do uso e aceitação de medicamentos genéricos pela população de Maringá-PR. Iniciação Científica Cesumar. v.7, n.2, p. 133-140, 2005.

PEREIRA, G.S. et al. Avaliação da utilização de medicamentos na prática clínica em um hospital público. Revista de Ciências Farmacêuticas Básica e Aplicada. v.32, n.2, p.239-244, 2011. 
SANTOS, V.; NITRINI, S.M.O.O. Indicadores do uso de medicamentos prescritos e de assistência ao paciente de serviços de saúde. Revista de Saúde Pública. v.38, n.6, p.819-834, 2004.

SILVA, J.L.; PIVA, C.; TESTON, A.P.M.; GRUEDELING, A.P.; FERREIRA, E.C.; TIYO, R. Avaliação da dispensação de medicamentos genéricos em farmácias privativas de Maringá - PR. Brazilian Journal of Surgery and Clinical Research. v.4, n.3, p.05-08, 2013.

SILVA, NCS; ROCHA, LC. Medicamentos genéricos: legislação, política e mercado. Única Cadernos Acadêmicos. v.3, n.2, 2016.

UTZIG, M.F.K.; VIRTUOSO, S.; DE OLIVEIRA, S.M.M. Adesão aos medicamentos genéricos: verificação preliminar após sete anos de implantação da lei. Visão Acadêmica. v.10, n.2, p.100105, 2009.

VALLÉS, J. et al. A perspective multicenter study of de effect of patient education on acceptability of generic prescribing in general pratice. Health Policy. v.65, n.3, p.269-275, 2003.

VIEIRA, F. S.; ZUCCHI, P. Diferenças de preço entre medicamentos genéricos e de referência no Brasil. Revista de Saúde Pública, v. 40, n. 3, p. 444-449, 2006.

WORLD HEALTH ORGANIZATION (WHO). Adherence to long-term therapies: evidence for action. Geneva, 2003. $199 \mathrm{p}$

WORLD HEALTH ORGANIZATION (WHO). Equitable access to essential medicines: a framework for collective action. Geneva: WHO; 2004b. Disponível em: <http://apps.who.int/medicinedocs/pdf/s4962e/s4962e.pdf>. Acessado em: 14 de maio de 2017. 